\title{
The OPERA experiment on the CNGS observed its first $v_{\tau}$ candidate
}

\author{
P. Migliozzi ${ }^{* \dagger}$ \\ INFN - Napoli \\ E-mail: migliozzi@na.infn.it
}

In this paper after a brief description of the beam, the experimental setup and the procedures used for the analysis of the neutrino events, we describe the topology and kinematics of a first candidate $v_{\tau}$ charged-current event satisfying the kinematical selection criteria. The background calculations and their cross-check are explained and the significance of the event is assessed. Finally, the present status and the outlook for the experiment are discussed.

35th International Conference of High Energy Physics - ICHEP2010,

July 22-28, 2010

Paris France

* Speaker.

†n behalf of the OPERA Collaboration. 


\section{Introduction}

Neutrino oscillations have been discovered almost 10 years ago by the SK [1]. Since then several experiments carried out in the last decades with atmospheric and accelerator neutrinos, as well as with solar and reactor neutrinos, have contributed to our present understanding of neutrino mixing (see e.g. [2] for a review). However, the direct observation of flavour transition through the detection of the corresponding lepton has never been observed. Appearance of $v_{\tau}$ will prove unambiguously that $v_{\mu} \rightarrow v_{\tau}$ oscillation is the dominant transition channel at the atmospheric scale. This is the main goal of the OPERA experiment $[3,4,5]$ with the high energy long baseline CNGS [6] neutrino beam from CERN to the Gran Sasso Underground Laboratory of INFN.

\section{The OPERA detector and the CNGS beam}

The OPERA experiment [7] aims at measuring the first detection of neutrino oscillation in appearance mode through the detection of $v_{\tau}$ in an almost pure $v_{\mu}$ beam produced at CERN SPS (CNGS), $730 \mathrm{~km}$ far from the detector. The $v_{\tau}$ appearance signal is detected through the measurement of the decay daughter particles of the $\tau$ lepton produced in CC $v_{\tau}$ interactions. Since the short-lived $\tau$ particle has, at the energy of the beam, an average decay length of about $1 \mathrm{~mm}$, a micrometric detection resolution is needed. In OPERA, neutrinos interact in a large mass target made of lead plates interspaced with nuclear emulsion films acting as high-accuracy tracking devices. This kind of detector is historically called Emulsion Cloud Chamber (ECC). OPERA is an hybrid detector [7] made of a veto plane followed by two identical Super Modules (SM) each consisting of a target section of about 625 tons made of 75000 emulsion/lead ECC modules or "bricks", of a scintillator Target Tracker detector (TT) to trigger the read-out and localize neutrino interactions within the target, and of a muon spectrometer. A target brick consists of 56 lead plates of $1 \mathrm{~mm}$ thickness interleaved with 57 emulsion films and weighs $8.3 \mathrm{~kg}$. Their thickness along the beam direction corresponds to about 10 radiation lengths. In order to reduce the emulsion scanning load, Changeable Sheets (CS) film interfaces have been used. They consist in tightly packed doublets of emulsion films glued to the downstream face of each brick.

Charged particles from a neutrino interaction in a brick cross the CS and produce signals in the TT that allow the corresponding brick to be identified and extracted by an automated system. Large ancillary facilities are used to bring bricks from the target up to the automatic scanning microscopes at LNGS and various laboratories in Europe and Japan [8, 9]. Extensive information on the OPERA detector and ancillary facilities are given in $[7,10]$.

The CNGS beam is optimized for the observation of $v_{\tau}$ CC interactions. The average neutrino energy is $\sim 17 \mathrm{GeV}$. The $\bar{v}_{\mu}$ contamination is $2.1 \%$ in terms of interactions; the $v_{e}$ and $\bar{v}_{e}$ contaminations are lower than $1 \%$, while the number of prompt $v_{\tau}$ is negligible. With a total CNGS beam intensity of $22.5 \times 10^{19}$ protons on target (p.o.t.), about 24300 neutrino events would be collected. We report here on results based on part of the data taken during 2008 and 2009 runs, which amounted respectively to 1.78 and $3.52 \times 10^{19}$ p.o.t., respectively. Among the 10122 and 21428 on-time events taken in 2008 and 2009, respectively 1698 and 3693 events were identified as candidate neutrino interactions in the target bricks. 

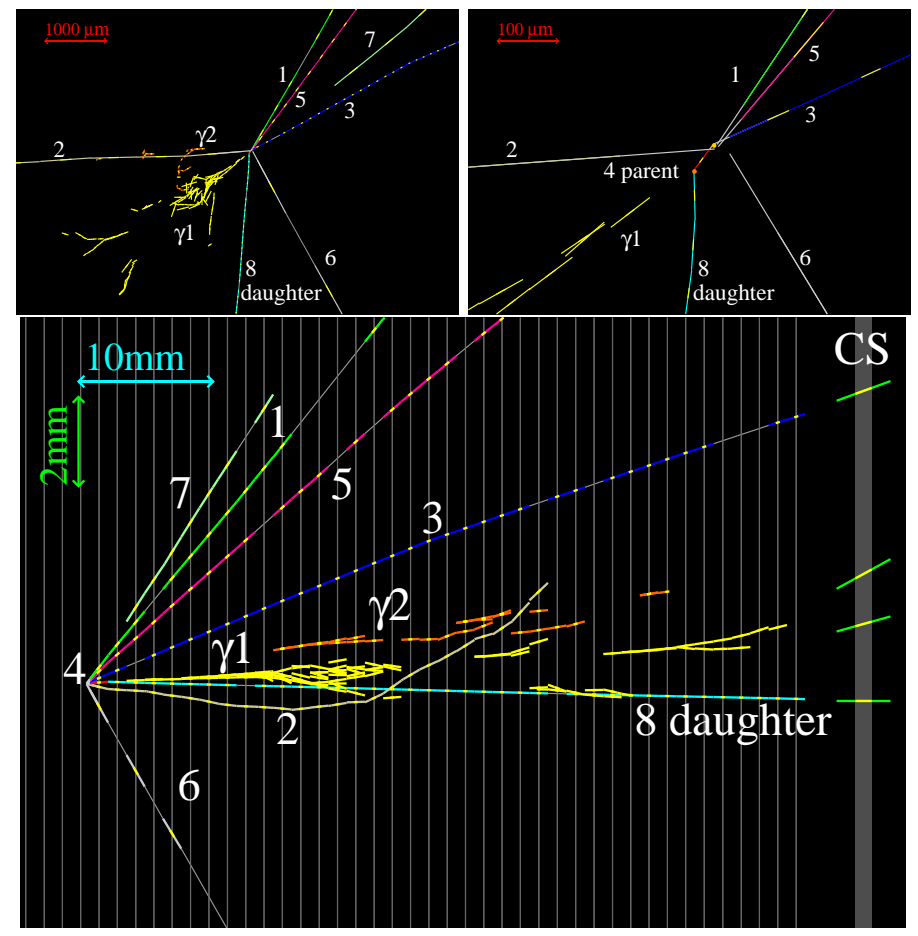

Figure 1: Display of the $\tau^{-}$candidate event. Top left: view transverse to the neutrino direction. Top right: same view zoomed on the vertices. Bottom: longitudinal view.

\section{Results}

In Ref [11] the OPERA Collaboration reported the observation of a first candidate $v_{\tau}$ CC interaction in the OPERA detector at LNGS. The primary neutrino interaction consists of 7 tracks of which one exhibits a visible kink. Two electromagnetic showers caused by $\gamma$-rays have been located that are associated with the event and in particular produced at the decay vertex. Fig. 1 shows a display of the event. It was identified in a sample of events corresponding to $1.89 \times 10^{19}$ p.o.t. in the CERN CNGS $v_{\mu}$ beam. The total transverse momentum $P_{T}$ of the daughter particles with respect to the parent track is $\left(0.47_{-0.12}^{+0.24}\right) \mathrm{GeV} / \mathrm{c}$, above the lower selection cut-off at $0.3 \mathrm{GeV} / \mathrm{c}$. The missing transverse momentum $P_{T}^{\text {miss }}$ at the primary vertex is $\left(0.57_{-0.17}^{+0.32}\right) \mathrm{GeV} / \mathrm{c}$. This is lower than the upper selection cut-off at $1 \mathrm{GeV} / \mathrm{c}$. The angle $\Phi$ between the parent track and the rest of the hadronic shower in the transverse plane is equal to $(3.01 \pm 0.03)$ rad, largely above the lower selection cut-off fixed at $\pi / 2$. The invariant mass of $\gamma$-rays is $(120 \pm 20$ (stat. $) \pm 35$ (syst. $)) \mathrm{MeV} / \mathrm{c}^{2}$, supporting the hypothesis that they originate from a $\pi^{0}$ decay. Similarly the invariant mass of the charged decay product assumed to be a $\pi^{-}$and of the two $\gamma$-rays is $\left(640_{-80}^{+125}(\text { stat. })_{-90}^{+100}(\right.$ syst. $\left.)\right) \mathrm{MeV} / \mathrm{c}^{2}$, which is compatible with the $\rho$ (770) mass. The branching ratio of the decay mode $\tau \rightarrow \rho^{-} v_{\tau}$ is about $25 \%$. The observation of one possible tau candidate in the decay channel $h^{-}\left(\pi^{0}\right) v_{\tau}$ has a significance of $2.36 \sigma$ of not being a background fluctuation (background in this channel $0.018 \pm 0.007$ ). If one considers all decay modes included in the search the significance of the observation becomes 2.01 $\sigma$, being the total background $0.045 \pm 0.023$. 


\section{Present status and outlook}

The OPERA experiment has been approved to take data till the end of 2012. By that time we expect to collected about $18 \times 10^{19}$ p.o.t., that corresponds to about $10 \tau$ events detected in the OPERA Emulsion Cloud Chambers. So far the 2008 statistics has been successfully analyzed, while we expect to complete the 2009 analysis by the end of 2010. The $\tau$ detected expected in the $2008+2009$ statistics is of about 2 events. The results of this analysis will be published by Spring 2011.

At the time this paper is prepared the 2010 CNGS run has been successfully completed and $4.04 \times 10^{19}$ p.o.t. have been collected, i.e. 4246 events identified as candidate neutrino interactions in the target bricks. It is worth noting that in 2010 the overall CERN accelerator complex and the CNGS beam efficiency has been $82.1 \%$.

\section{References}

[1] SUPER-KAMIOKANDE collaboration, Y. Fukuda et al., Phys. Rev. Lett. 81 (1998) 1562; SUPER-KAMIOKANDE collaboration, K. Abe et al., Phys. Rev. Lett. 97 (2006) 171801; SUPER-KAMIOKANDE collaboration, R. Wendell et al., arXiv:1002.3471.

[2] C. Amsler et al. (Particle Data Group), Phys. Lett. B 667 (2008) 1; See 2009 updates at http://pdg.web.cern.ch/pdg/

[3] A. Ereditato, K. Niwa and P. Strolin, The emulsion technique for short, medium and long baseline $v_{\mu} \rightarrow v_{\tau}$ oscillation experiments, 423, INFN-AE-97-06, DAPNU-97-07;

OPERA collaboration, H. Shibuya et al., Letter of intent: the OPERA emulsion detector for a long-baseline neutrino-oscillation experiment, CERN-SPSC-97-24, LNGS-LOI-8-97.

[4] OPERA collaboration, M. Guler et al., An appearance experiment to search for $v_{\mu} \rightarrow v_{\tau}$ oscillations in the CNGS beam: experimental proposal, CERN-SPSC-2000-028, LNGS P25/2000

[5] OPERA collaboration, M. Guler et al., Status Report on the OPERA experiment, CERN/SPSC 2001-025, LNGS-EXP 30/2001 add. 1/01

[6] Ed. K. Elsener, The CERN Neutrino beam to Gran Sasso (Conceptual Technical Design), CERN 98-02, INFN/AE-98/05;

R. Bailey et al., The CERN Neutrino beam to Gran Sasso (NGS) (Addendum to report CERN 98-02, INFN/AE-98/05), CERN-SL/99-034(DI), INFN/AE-99/05.

[7] R. Acquafredda et al. [ OPERA Collaboration ], JINST 4 (2009) P04018.

[8] N. Armenise et al., Nucl. Instrum. Meth. A 551 (2005) 261;

M. De Serio et al., Nucl. Instrum. Meth. A 554 (2005) 247;

L. Arrabito et al., Nucl. Instrum. Meth. A 568 (2006) 578.

[9] K. Morishima and T. Nakano, JINST (2010) 5 P04011.

[10] OPERA collaboration, A. Anokhina et al., JINST 3 (2008) P07002; OPERA collaboration, A. Anokhina et al., JINST 3 (2008) P07005;

T. Adam et al., Nucl. Instrum. Meth. A 577 (2007) 523;

T. Nakamura et al., Nucl. Instrum. Meth. A 556 (2006) 80.

[11] N. Agafonova et al. [ OPERA Collaboration ], Phys. Lett. B691 (2010) 138-145. [arXiv:1006.1623 [hep-ex]]. 